\title{
RIGID LEFT NOETHERIAN RINGS
}

\section{O. D. ARTEMOVYCH}

Received 17 January 2003

We prove that any rigid left Noetherian ring is either a domain or isomorphic to some ring $\mathbb{Z}_{p^{n}}$ of integers modulo a prime power $p^{n}$.

2000 Mathematics Subject Classification: 16P40, 16W20, 16W25.

Let $R$ be an associative ring. A map $\sigma: R \rightarrow R$ is called a ring endomorphism if $\sigma(x+y)=\sigma(x)+\sigma(y)$ and $\sigma(x y)=\sigma(x) \sigma(y)$ for all elements $a, b \in R$. A ring $R$ is said to be rigid if it has only the trivial ring endomorphisms, that is, identity $\mathrm{id}_{R}$ and zero $0_{R}$. Rigid left Artinian rings were described by Maxson [9] and McLean [11]. Friger [4, 6] has constructed an example of a noncommutative rigid ring $R$ with the additive group $R^{+}$of finite Prüfer rank. A characterization for rigid rings of finite rank was obtained by the author in [1]. Some aspects of a ring rigidity has been studied by Suppa [12, 13], Friger [5], and the author [2].

In this paper, we study rigid left Noetherian rings and prove the following theorem.

THEOREM 1. Let $R$ be a left Noetherian ring. Then $R$ is a rigid ring if and only if $R \cong \mathbb{Z}_{p^{t}}$ ( $p$ is a prime, $t \in \mathbb{N}$ ) or it is a rigid domain.

All rings are assumed to be associative and, as a rule, with an identity element. For a ring $R, N(R)$ will always denote the set of all nil elements of $R$, $\operatorname{char}(R)$ the characteristic, and $\operatorname{Ann}(I)=\{a \in R \mid a I=I a=\{0\}\}$ the annihilator of $I$ in $R$. If $R$ is a left order in $Q$ (or equivalently, $Q$ is the left quotient ring of $R$ ), then we will write $Q=Q(R)$. Any unexplained terminology is standard as in [10].

We recall that a ring $R$ is reduced if $r^{2}=0$ implies $r=0$ for any $r \in R$. Clearly, if $R$ is a rigid reduced ring with an identity element, then either $\operatorname{char}(R)=0$ or $\operatorname{char}(R)=p$ for some prime $p$.

LEMMA 2. Let $R$ be a reduced left Goldie ring. If $R$ is rigid, then it is a domain.

Proof. Let $R$ be a reduced rigid left Goldie ring. Assume that $R$ is not a domain. From $b x=0$ (resp., $x b=0$ ), where $b, x \in R$, it holds that $(x b)^{2}=0$ (resp., $(b x)^{2}=0$ ) and thus a right (resp., left) annihilator of every element $b$ in $R$ coincides with Ann $(b)$. Moreover, in view of [10, Lemma 2.3.2(i)], Ann $(a)$ is a maximal left annihilator for some $a \in R$.

Assume that the quotient ring $R / \operatorname{Ann}(a)$ contains elements $\bar{x}=x+\operatorname{Ann}(a) \neq \overline{0}$, $\bar{y}=y+\operatorname{Ann}(a)$ such that

$$
\bar{x} \bar{y}=\overline{0}
$$


for some $x, y \in R$. Since $y \in \operatorname{Ann}(a x)$ and $\operatorname{Ann}(a)=\operatorname{Ann}(a x)$, we obtain that $\bar{y}=\overline{0}$. This means that $R / \operatorname{Ann}(a)$ is a domain.

By [10, Lemma 2.3.3], $I_{a}=R a \oplus \operatorname{Ann}(a)$ is an essential left ideal of $R$ and so by [10, Corollary 3.1.8], $Q\left(I_{a}\right)=Q(R)$. Then the map $\sigma: I_{a} \rightarrow I_{a}$ given by $\sigma(r a)=r a(r \in R)$ and $\sigma(\operatorname{Ann}(a))=\{0\}$ is a nontrivial ring endomorphism of $I_{a}$. If $\bar{\sigma}: Q(R) \rightarrow Q(R)$ is an extension of $\sigma$ to $Q(R)$, then

$$
\bar{\sigma}(r) a=\bar{\sigma}(r a)=r a
$$

for any $r \in R$, in which case,

$$
a(\bar{\sigma}(r)-r)=0=(\bar{\sigma}(r)-r) a .
$$

Since $\bar{\sigma}(r)-r=q^{-1} t$ for some regular element $q \in R$ and some $t \in R$, we see that

$$
q(\bar{\sigma}(r)-r) \in \operatorname{Ann}(a)
$$

But $q \notin \operatorname{Ann}(a)$ and so $\bar{\sigma}(r)-r \in \operatorname{Ann}(a)$. This means that $\bar{\sigma}(R) \subseteq R$ and $R$ has a nontrivial ring endomorphism, a contradiction. The lemma is proved.

In the commutative case, we obtain that a commutative reduced rigid Noetherian ring $R$ of finite exponent is isomorphic to some $\mathbb{Z}_{p}$.

Indeed, as it is noted above, $\operatorname{char}(R)=p$ for some prime $p$. A map $\omega: R \rightarrow R$ given by the rule $\omega(x)=x^{p}(x \in R)$ is a ring endomorphism of $R$ and so $x^{p}=x$ for all elements $x$ of $R$. Assume that $R$ is not a domain and then it follows that every prime ideal is maximal in $R$. Hence $R$ is an Artinian ring by Krull-Akizuki theorem [14, Chapter IV, Section 2, Theorem 2] and by the theorem of [11], $R \cong \mathbb{Z}_{p}$, contrary to our assumption. This means that $R$ is a domain and [9, Theorem 2.5] allows us to state that $R \cong \mathbb{Z}_{p}$.

REMARK 3. Maxson [9] has proved that a rigid commutative domain of prime characteristic $p$ is isomorphic to $\mathbb{Z}_{p}$. Rigid rings of finite rank were studied in [1]. A characterization of rigid commutative domains (in particular, rigid fields) $R$ of characteristic 0 with the additive group $R^{+}$of infinite (Prüfer) rank is not known. As it is noted in [8], from the result of Gaifman [7], it holds that there exist rigid Peano fields of arbitrary infinite cardinality. Moreover, it was proved by Dugas and Göbel [3] that each field can be embedded into a rigid field of arbitrary large cardinality.

REMARK 4. There exist noncommutative rigid Noetherian domains of characteristic 0 (see $[4,6])$.

Recall that a map $d: R \rightarrow R$ is called a derivation of $R$ if

$$
d(x+y)=d(x)+d(y), \quad d(x y)=d(x) y+x d(y)
$$


for all elements $x, y \in R$. A ring having no nonzero derivations is called differentially trivial (see [1]). Obviously, any differentially trivial ring is commutative.

LEMMA 5. Let $R$ be a left Noetherian ring such that $N(R) \neq\{0\}$. If $R$ is a rigid ring, then it is isomorphic to some $\mathbb{Z}_{p^{t}}$.

Proof. Suppose that $R$ is a rigid ring such that $N=N(R) \neq\{0\}$. Then $N \subseteq Z(R)$ (see [9, page 96]). Let $d$ be any nonzero derivation of $R$. If $z d(R)=\{0\}$ for all elements $z \in N$ of the nilpotency indices $i<n-1$ and $a d(R) \neq\{0\}$ for some element $a \in N$ of the nilpotency index $n$, then the rule

$$
\sigma(r)=r+a d(r), \quad r \in R
$$

determines a nontrivial ring endomorphism $\sigma$ of $R$, a contradiction. Hence

$$
N(R) d(R)=\{0\}
$$

for every derivation $d$ of $R$.

Let $K_{0}=\left\{a \in N \mid\left(N \cap \operatorname{Ann}\left(N^{2}\right)\right) a=\{0\}\right\}$. Then $N \cap \operatorname{Ann}\left(K_{0}\right)=N \cap \operatorname{Ann}\left(N^{2}\right)$. Assume that $\delta: R / K_{0} \rightarrow R / K_{0}$ is a nonzero derivation of $R / K_{0}$ and therefore for every $r \in R$, there is an element $r_{1} \in R$ such that

$$
\delta\left(r+K_{0}\right)=r_{1}+K_{0}
$$

Moreover, $a_{1} \notin K_{0}$ for some $a \in R$. Writing $I$ for the two-sided ideal of $R$ generated by $a_{1}$, we see that $\left(N \cap \operatorname{Ann}\left(N^{2}\right)\right)\left(K_{0}+I\right) \neq\{0\}$. Thus there exists an element $m_{0} \in$ $N \cap \operatorname{Ann}\left(N^{2}\right)$ such that $m_{0} a_{1} \neq 0$ and so the rule $g(r)=m_{0} r_{1}$, with $r \in R$ and $r_{1}$ as in (8), determines a nonzero derivation $g$ of $R$. In view of $(7) g(r) g(t)=0$, for any elements $r, t \in R$ and a map $\alpha: R \rightarrow R$ given by the rule $\alpha(r)=r+g(r),(r \in R)$ is a nontrivial ring endomorphism of $R$, a contradiction with hypothesis. This gives that $R / K_{0}$ is differentially trivial and consequently commutative. Since $K_{0} \subseteq N$ and $N \subseteq Z(R)$, $R$ is a Noetherian ring and, as a consequence of [10, Theorem 4.1.9] and [9, Theorem 2.2], $R$ is an Artinian ring. Finally, by the theorem from [11], $R \cong \mathbb{Z}_{p^{t}}$ for some prime $p$ and integer $t$. This completes the proof.

Proof of TheOrem 1. It follows immediately from Lemmas 2 and 5.

COROLlary 6. Any rigid simple left Goldie ring $R$ is a field (or equivalently, any noncommutative simple left Goldie ring has a nontrivial automorphism).

Proof. Since $N(R) \subseteq Z(R), R$ is a semiprime ring and so according to [10, Proposition 5.1.5] and Lemma 2, it is a domain. If $q$ is any element of $Q(R) \backslash R$ and $A=q^{-1} R q$, then $A$ is a left order in $Q(R)$. Moreover, $q A q^{-1}=R$ and so $A$ and $R$ are equivalent left orders in $Q(R)$. By [10, Proposition 5.1.2], $R$ is a maximal left order in $Q(R)$ and thus $A \subseteq R$, which implies $R \subseteq Z(Q(R))$, as required. 


\section{REFERENCES}

[1] O. D. Artemovych, Differentially trivial and rigid rings of finite rank, Period. Math. Hungar. 36 (1998), no. 1, 1-16.

[2] _ On I-rigid and q-rigid rings, Ukraïn. Mat. Zh. 50 (1998), no. 7, 989-993 (Russian).

[3] M. Dugas and R. Göbel, All infinite groups are Galois groups over any field, Trans. Amer. Math. Soc. 304 (1987), no. 1, 355-384.

[4] M. D. Friger, Rigid torsion-free rings, Sibirsk. Mat. Zh. 27 (1986), no. 3, 217-219 (Russian).

[5] _ Strongly rigid and I-rigid rings, Comm. Algebra 22 (1994), no. 5, 1833-1842.

[6] _ Torsion-free rings: some results on automorphisms and endomorphisms, Second International Conference on Algebra (Barnaul, 1991), Contemp. Math., vol. 184, American Mathematical Society, Rhode Island, 1995, pp. 111-115.

[7] H. Gaifman, Models and types of Peano's arithmetic, Ann. Math. Logic 9 (1976), no. 3, 223306.

[8] C. U. Jensen and H. Lenzing, Model-Theoretic Algebra with Particular Emphasis on Fields, Rings, Modules, Algebra, Logic and Applications, vol. 2, Gordon and Breach Science Publishers, New York, 1989.

[9] C. J. Maxson, Rigid rings, Proc. Edinburgh Math. Soc. (2) 21 (1979), no. 2, 95-101.

[10] J. C. McConnell and J. C. Robson, Noncommutative Noetherian Rings, Pure and Applied Mathematics, John Wiley \& Sons, Chichester, 1987.

[11] K. R. McLean, Rigid Artinian rings, Proc. Edinburgh Math. Soc. (2) 25 (1982), no. 1, 97-99.

[12] M. A. Suppa, Sugli anelli I-rigidi [On I-rigid rings], Boll. Un. Mat. Ital. D (6) 4 (1985), no. 1, 145-152 (Italian).

[13] _ Sugli anelli q-rigidi, Riv. Mat. Univ. Parma 12 (1986), 121-125 (Italian).

[14] O. Zariski and P. Samuel, Commutative Algebra. Vol. II, The University Series in Higher Mathematics, D. Van Nostrand C., New Jersey, 1960.

O. D. Artemovych: Institute of Mathematics, Cracow University of Technology, Warszawska 24, Cracow 31-155, Poland

E-mail address: artemo@usk.pk.edu.p1 


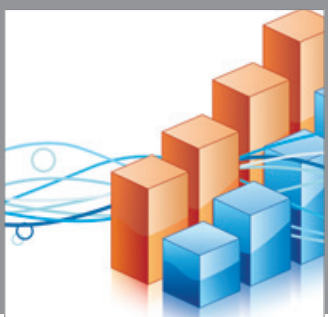

Advances in

Operations Research

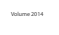

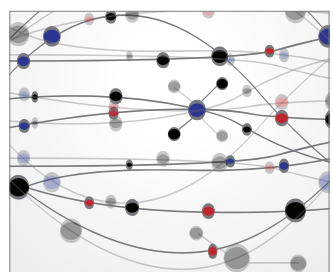

\section{The Scientific} World Journal
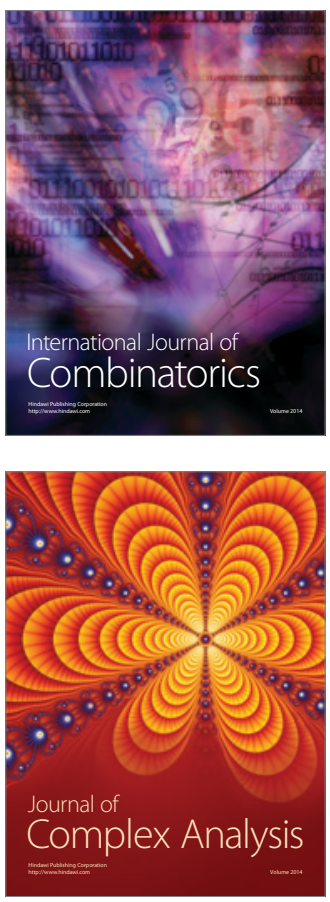

International Journal of

Mathematics and

Mathematical

Sciences
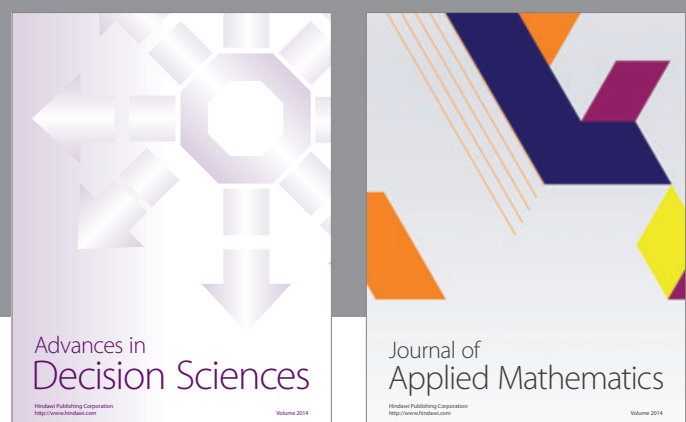

Journal of

Applied Mathematics
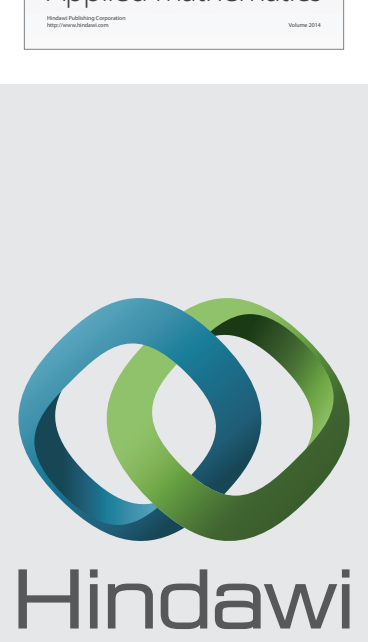

Submit your manuscripts at http://www.hindawi.com
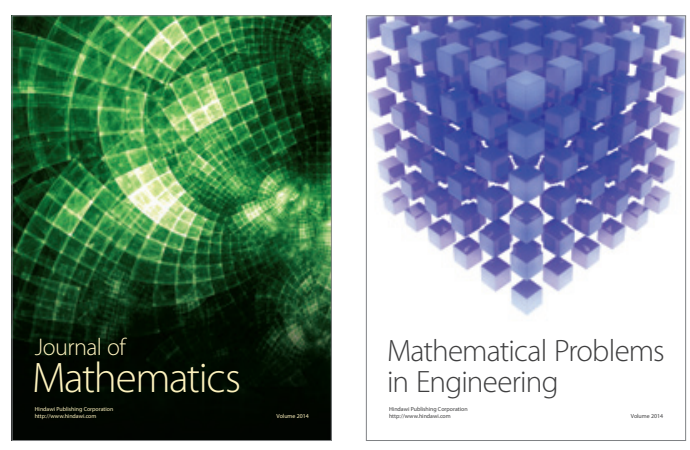

Mathematical Problems in Engineering
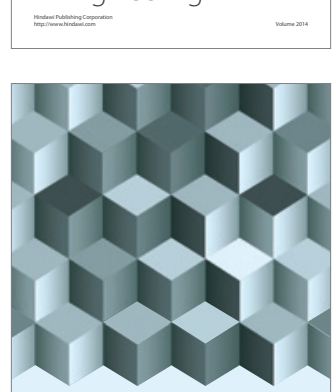

Journal of

Function Spaces
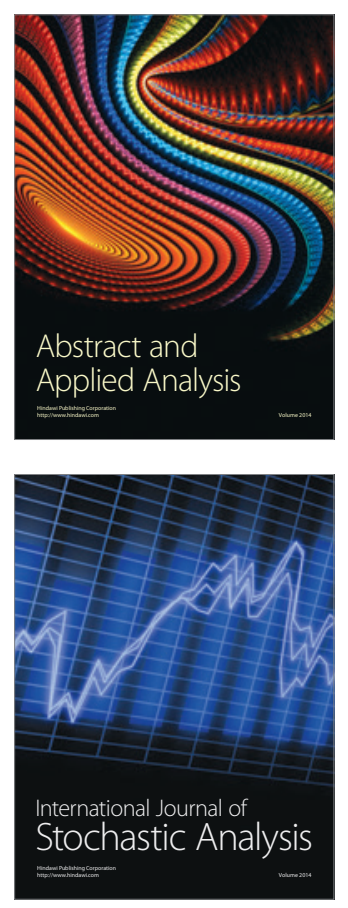

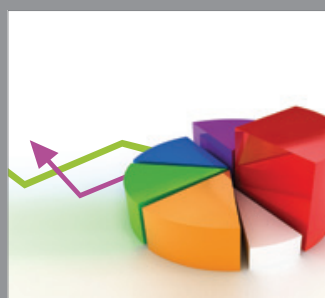

ournal of

Probability and Statistics

Promensencen
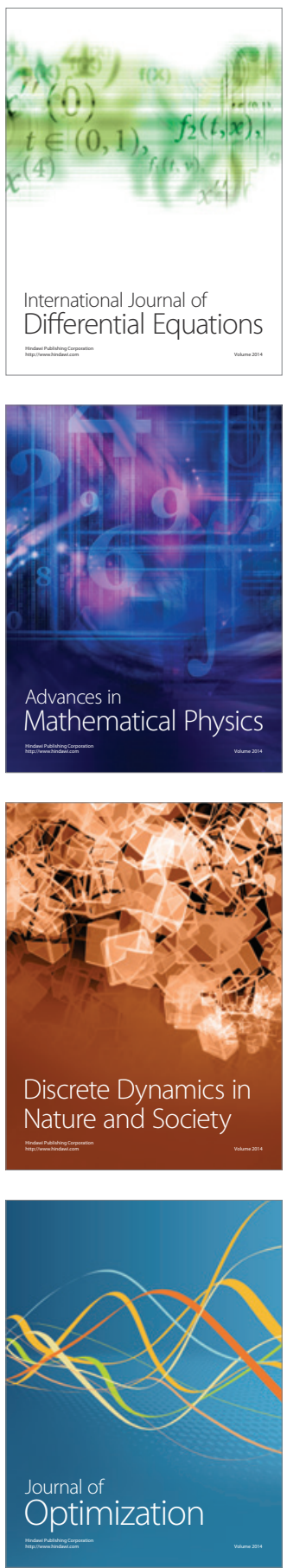